\title{
CMS tracking challenges yesterday, today and tomorrow
}

\section{Erica Brondolin*}

Institut fur Hochenergiephysik der OeAW (AT)

E-mail: erica.brondolinecern.ch

The Compact Muon Solenoid (CMS) is one of the two general purpose experiments at the Large Hadron Collider at CERN. Until 2012 (RunI), pp collisions have been delivered with a minimal bunch time separation of $50 \mathrm{~ns}$ and a mean of about 15 collisions per bunch crossing. After the end of the long shut-down this year, LHC is foreseen to ultimately exceed an instantaneous luminosity of $10^{34} \mathrm{~cm}^{-2} \mathrm{~s}^{-1}$, at a bunch time separation of $25 \mathrm{~ns}$ with a mean of more than 25 inelastic collisions superimposed on the event of interest (RunII). In this high-occupancy environment, obtaining a precise particle momentum reconstruction is one of the biggest challenges. To this end, the CMS collaboration has constructed the largest silicon tracker ever and has developed a sophisticated tracking software, that is able to successfully reconstruct the hundreds of tracks produced in each beam crossing. However, more challenges lie ahead. CERN is planning an upgrade program of LHC which will increase the luminosity to $5 \times 10^{34} \mathrm{~cm}^{-2} \mathrm{~s}^{-1}$ after 2020 . In order to face this new scenario (called PhaseII), CMS will build a completely new silicon tracker and will need to implement new approaches to track finding in addition to the algorithms already in use. This paper gives an overview of the iterative track reconstruction used in CMS with the performance obtained yesterday (RunI), recent tracking improvements for today (RunII), and some ideas (and projections) for tomorrow (PhaseII).

The European Physical Society Conference on High Energy Physics

22-29 July 2015

Vienna, Austria

${ }^{*}$ Speaker. 


\section{Yesterday: tracking performance in RunI}

From 2010 to 2012, the Large Hadron Collider (LHC) operated with an instantaneous luminosity up to $3.9 \times 10^{33} \mathrm{~cm}^{-2} \mathrm{~s}^{-1}$ and with the proton bunches crossing at intervals of $50 \mathrm{~ns}$. In these conditions, the Compact Muon Solenoid (CMS) tracker is expected to be crossed by about 1000 charged particles per bunch crossing, produced by 15 collisions on average. The interactions superimposed to the interesting event, called pile-up, is one of the most serious difficulties for the experimental operation at the LHC. In order to obtain a precise and efficient measurement of charged particles in this environment, CMS features the largest all-silicon tracker ever built. It is completely immersed in a 3.8 T magnetic field and consists of a small silicon pixel tracker close to the interaction point, and a large silicon strip tracker surrounding it (Figure 1). It covers the range of pseudorapidity up to $|\eta|=2.5$ [1].

The pixel detector is located at a radial distance of less than $10 \mathrm{~cm}$ from the beamline, where the rate of particles is about $10^{7} \mathrm{~cm}^{-2} \mathrm{~s}^{-1}$. It has 66 million pixels and covers a total area of about $1.6 \mathrm{~m}^{2}$. It is arranged in three barrel layers and four disks. In the barrel, the resolution of a single hit is $\simeq 10 \mu \mathrm{m}$ in the $(r, \phi)$ coordinate and $\simeq 15 \mu \mathrm{m}$ in the $z$ coordinate, while in the endcaps it is $\simeq 15 \mu \mathrm{m}$ and $20-40 \mu \mathrm{m}$, respectively. The silicon strip tracker constitutes the outer part of the tracking system with 10 barrel detection layers extending up to a radius of $1.1 \mathrm{~m}$ and with two blocks of forward disks. The hit resolution is $\simeq 40 \mu \mathrm{m}$ in $(r, \phi)$. The CMS silicon strip tracker has a total of 9 million strips and an active silicon area of about $200 \mathrm{~m}^{2}$. In some layers modules are made of double-sided strip sensors, one of which is rotated by a stereo angle, achieving a resolution along the $z$ coordinate of about $\simeq 230 \mu \mathrm{m}$ and in this way, allowing the reconstruction of the hit position in 3-D (blue modules in Figure 1).

A detailed description of the software algorithms developed for the CMS track reconstruction and the results on performance obtained for RunI with a centre-of-mass energy of $\sqrt{s}=7 \mathrm{TeV}$ are given in Ref. [2]. The track finding proceeds in several iterations. The basic idea is to search for the tracks with higher transverse momentum first, to mask the hits associated to the High Purity ones (defined in Ref. [2]) and to continue with the next iteration. In this way the combinatorial

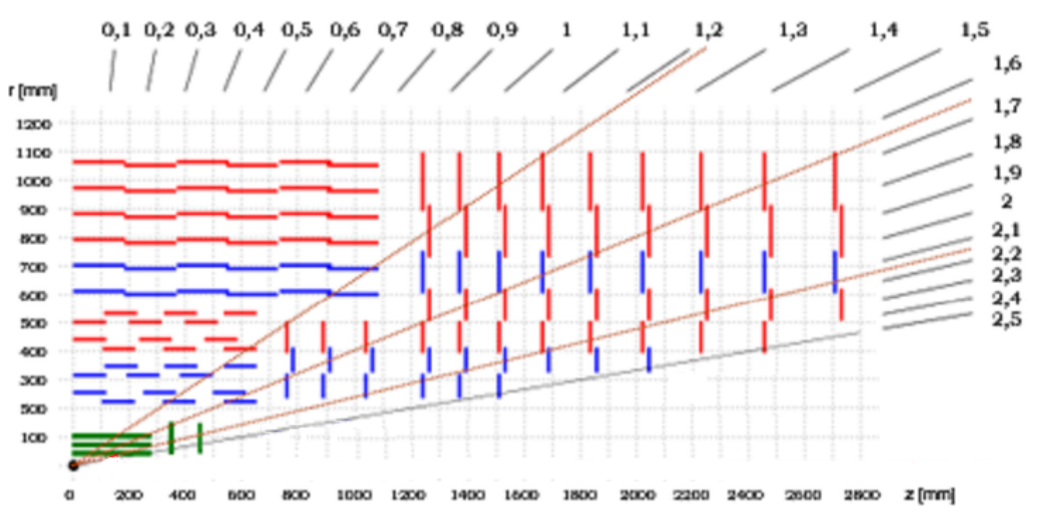

Figure 1: Schematic section through the CMS tracker. The innermost part is composed by pixel modules (green), while the outermost part is composed by two different kind of strip modules that can provide 3-D (blue) and 2-D hits (red). 


\begin{tabular}{cccc}
\hline \hline Iteration & Step Name & Seeding & Target Track \\
\hline 0 & Initial & pixel triplets & prompt, high $p_{\mathrm{T}}$ \\
1 & LowPtTriplet & pixel triplets & prompt, low $p_{\mathrm{T}}$ \\
2 & PixelPair & pixel pairs & high $p_{\mathrm{T}}$ recovery \\
3 & DetachedTriplet & pixel triplets & displaced \\
4 & MixedTriplet & pixel+strip triplets & displaced \\
5 & PixelLess & inner strip pairs & displaced \\
6 & TobTec & outer strip pairs & displaced \\
\hline
\end{tabular}

Table 1: List of different tracking iterations used in RunI with the corresponding seeding configuration used and target tracks.

complexity of the tracking problem is reduced and simplified for more difficult classes of tracks. Each iteration can be divided in four different steps:

1. Seed generation: Using only two or three hits, it provides the initial track candidates and the trajectory parameters and their uncertainties.

2. Track finding: It extrapolates the current trajectory parameters to the next layer and finds possible compatible hits which are then included into the track candidate. This step continues until there are no more layers or more than one hit is missing.

3. Track fitting: It performs a final Kalman or Gaussian sum filter/smoother in order to obtain the best possible estimation of the trajectory parameters at the interaction point.

4. Track selection: Track quality cuts are used to set the quality flags: Loose, Tight or High Purity.

In Table 1 the seven tracking iterations are listed along with the corresponding seeding configuration used and the target tracks. Figure 2 shows the excellent performance obtained in RunI. For $t \bar{t}$ events simulated with and without pile-up the efficiency is around $90 \%$ while keeping a low rate of fake tracks, i.e. combinations of unrelated hits.

\section{Today: new developments and results for Run2}

After the end of the long shut-down in 2015, LHC is foreseen to achieve the nominal instantaneous luminosity of $10^{34} \mathrm{~cm}^{-2} \mathrm{~s}^{-1}$ and the nominal centre-of-mass energy of $14 \mathrm{TeV}$. The bunch time separation foreseen is $25 \mathrm{~ns}$ with an average of 25 pile-up interactions. Higher pile-up and the subsequent rise in occupancy in the tracker is a challenge for tracking because of its intrinsic combinatorial nature. Several problems are raised by this situation: first, the fraction of unmasked hits after all iterations remains above 50\%; second, there is a dramatic timing effect on the iterations seeded by pairs in the double-sided strip modules, because of ghost hits (Figure 3(a)); third, the pixel detector is affected by dynamic inefficiency which is mainly due to saturation of the chip 


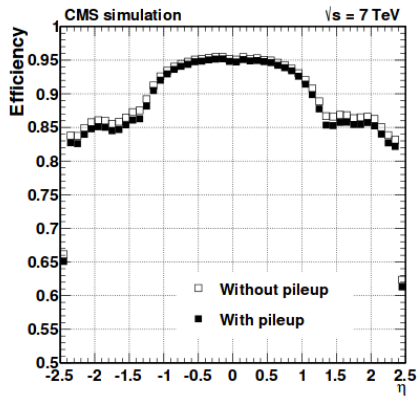

(a)

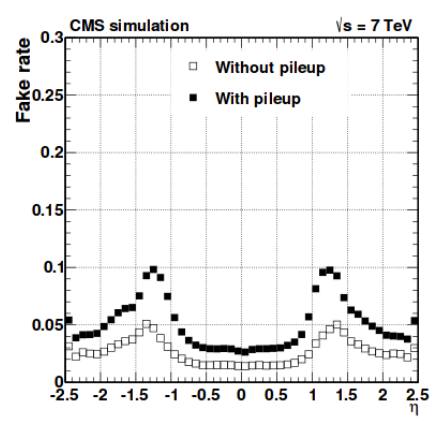

(b)

Figure 2: Tracking efficiency (a) and fake rate (b) for $t \bar{t}$ events simulated with and without superimposed pileup collisions. The number of pileup interactions superimposed on each simulated event is generated randomly from a Poisson distribution with a mean value of 8 [2].

readout buffer and reaches the $2 \%$ level already below the nominal instantaneous luminosity in the first pixel barrel layer.

In order to cope with these issues, some developments have been done in different aspects of the tracking procedure [3]:

- Two new iterations dedicated exclusively to muons have been added: an outside-in track reconstruction step seeded in the muon system, and an inside-out iteration that re-reconstructs muon-tagged tracks with looser requirements. The muon-track efficiency loss seen in 2012 for high pile-up is fully recovered, the muon momentum is more precise, and the identification is more robust.

- Some progress in the reconstruction of tracks inside jet cones has been made by introducing a new iteration, which searches for tracks around the direction of the high $p_{\mathrm{T}}$ calorimeter jets, and an iterative pixel cluster splitting. Thanks to these two developments, the track reconstruction efficiency inside jet cores has risen by about 20\% (Figure 3(b)).

- A new seeding algorithm dedicated to strips triplets has been developed using a straight line fit and the dynamic re-estimation of the hit positions using the seed direction hypothesis. This allows to reject half of the seeds, while reconstructing the same number of tracks (Figure $3(\mathrm{c}))$.

- Another development concerning mainly the strip-seeded iterations is the introduction of a cut on the cluster charge in order to reject the clusters produced by event overlapping between successive bunch crossing. It accounts both for sensor thickness and trajectory crossing angle and it can be applied with increasing thresholds at different stages of the track reconstruction. The fake rate is reduced by $\simeq 50 \%$ by this cut.

- Many other improvements have been realized: the code and the iterative tracking logic have been globally optimized, the track selection step now uses a multivariate technique, the pixel 


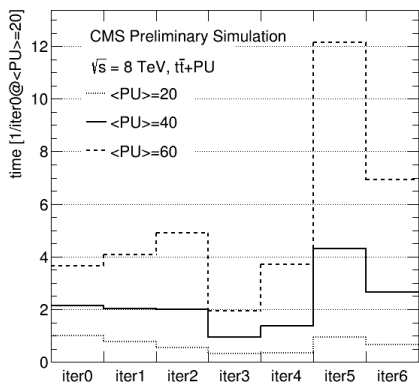

(a)

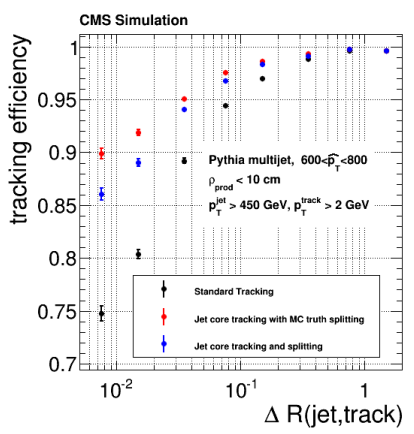

(b)

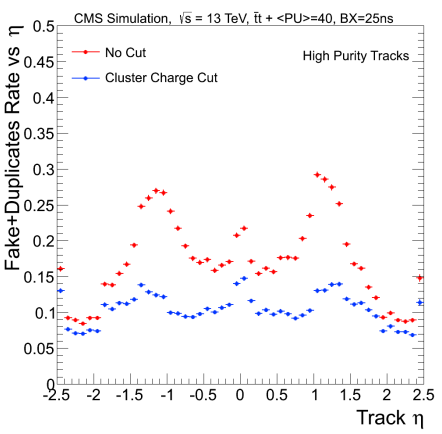

(c)

Figure 3: (a) Reconstruction time for each iterative tracking steps for $t \bar{t}$ sample with average pileup of 20, 40 or 60, normalized to the time of the first iteration. (b) Track reconstruction efficiency in multijet events as function of the $\Delta \mathrm{R}$ between the track and the jet axis for standard tracking (black), jet core tracking with a cluster splitting based on MC truth (red), and jet core tracking with new splitting algorithm (blue). (c) Tracking fake rate vs $\eta$ with (blue) and without (red) a selection on the strip cluster charge [3].

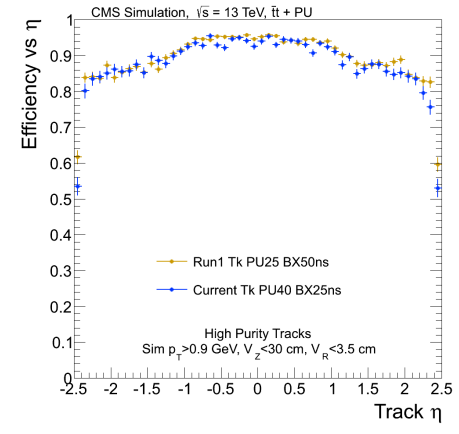

(a)

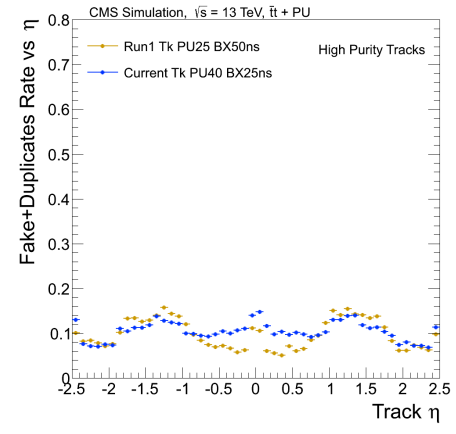

(b)

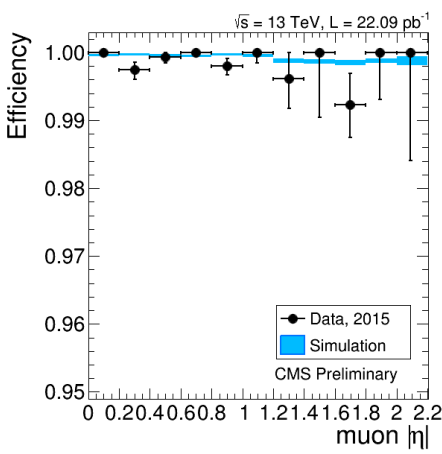

(c)

Figure 4: $(a, b)$ Comparison of tracking efficiency and the fake+duplicate rate for Run 1 and Run 2 typical pile-up conditions (25 and 40 respectively) [3]. (c) Comparison between early 2015 data and simulation tracking efficiency for muons is shown as a function of $|\eta|$ measured with the tag and probe method using $\mathrm{Z}$ bosons decaying into muon pairs [4].

dynamic inefficiency has been included in the simulation, and the High Level Trigger tracking software has been speeded up by a factor of 3.5.

In Figure 4(a,b) the efficiency and the fake rate for high $p_{\mathrm{T}}$ prompt tracks are shown in nominal conditions (RunI-like for RunI release, RunI-like for current release). The performances are very similar, so it is expected that in RunII CMS will be able to maintain the high tracking performance shown in RunI, but in a much more challenging environment. This has already been confirmed by the first results obtained with the new early data recorded: Figure 4(c) shows the excellent muon-track efficiency for both data and simulation. 


\section{Tomorrow: CMS tracking in PhaseII conditions}

Starting in 2023, LHC will be upgraded to the High Luminosity LHC (HL-LHC) in order to achieve a luminosity of $5 \times 10^{34} \mathrm{~cm}^{-2} \mathrm{~s}^{-1}$ with a bunch spacing of $25 \mathrm{~ns}$, corresponding to more than 100 pile-up events per bunch crossing. In Ref. [5] a detailed description of the entire PhaseII upgrade of the CMS experiment is presented. In particular, the rise of the LHC luminosity requires a substantial upgrade of the CMS tracking system (Figure 5(a)). The pixel system will have four barrel layers and 8-10 endcap disks, while the silicon strip tracker will have six barrel layers and five endcap disks. The new tracker will cover the range of pseudorapidity up to $|\eta|=4$. The main goals of the new silicon strip tracker will be: resisting the higher radiation level, keeping the channel occupancy low, and contributing to the Level-1 trigger. This last requirement is needed in the HL-LHC era because the trigger system is exploring the possibility to maintain a total output rate varying from $100 \mathrm{kHz}$ to $1 \mathrm{MHz}$ despite the tenfold luminosity. This appears to be impossible to achieve using only the information coming from the calorimeters and the muon detectors.

The CMS collaboration has therefore decided to introduce a L1 track trigger by designing modules that are able to reject low- $p_{\mathrm{T}}$ particles given the strong bending power of the $3.8 \mathrm{~T}$ magnetic field. This module will be a stack of two closely spaced sensors and will be able to correlate hits produced in the two layers in real time. In Figure 5(b) two reconstructed clusters in such a module are shown: particles with $p_{\mathrm{T}}$ higher than a certain threshold create a "stub" (left), instead particles with lower $p_{\mathrm{T}}$ are rejected (right). The $p_{\mathrm{T}}$ threshold of $2 \mathrm{GeV}$ reduces the data volume by about an order of magnitude, allowing to maintain an acceptable level of the global L1 trigger. Different kind of modules for the final configuration of the strip tracker are under study. One option includes in the inner part Macro Pixel-Strip (PS) modules composed of a bottom sensor with $1.5 \mathrm{~mm} \times 100 \mu \mathrm{m}$ pixels and a top sensor with $2 \times 25 \mathrm{~mm}$ strips $(100 \mu \mathrm{m}$ pitch $)$, and in the outer part Strip-Strip (2S) modules composed of two sensors with $2 \times 5 \mathrm{~cm}$ long strips ( $90 \mu \mathrm{m}$ pitch). In Figure 5(a) one possible arrangement of the foreseen 15508 new modules is shown, with a total active surface of $218 \mathrm{~m}^{2}$.

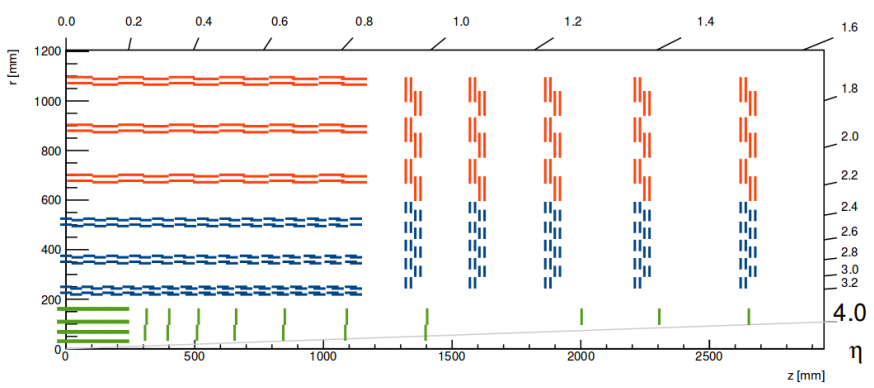

(a)

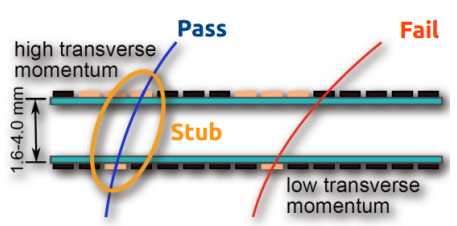

(b)

Figure 5: (a) Schematic section through the new CMS tracker foreseen for HL-LHC. The innermost part is composed of pixel modules (green), while the outermost part is composed by two different kind of strip modules: PS modules (blue) and $2 \mathrm{~S}$ modules (red). (b) The correlation of signals in closely-spaced sensors allows to identify "stub" produced by particles with $p_{\mathrm{T}}$ higher than a certain threshold and reject low- $p_{\mathrm{T}}$ particles. 
The combination of stubs in the different layers will provide the L1 track trigger primitives. Three different tracking approaches are being pursued by different teams:

- Associative Memory: The possible track candidates are found in a very fast way by performing the pattern recognition with a match against pre-computed track patterns (around 100 million reference patterns are needed for the full strip tracker). Different algorithms are under study for the track fit which is performed in a second step.

- Time Multiplexed Trigger: All data from a single event are sent through a single data processing module for assembly and processing. The Hough transform approach has been proposed to reconstruct track candidates.

- Tracklet approach: FPGAs are used to implement the traditional Kalman Filter road search where the track primitives considered are tracklets, obtained by linking up stubs.

A preliminary result on the resolution of the impact parameter using the third approach is shown in Figure 6(a). In Ref. [5], also the expected performance of the offline tracking for the upgraded detector is presented. The current PhaseII tracking is based on PhaseI track reconstruction adapted to a pile-up around 140: six iterations with pixel seeding and two iterations dedicated to muon search. In Figure 6(b) the efficiency as a function of the pseudorapidity for both PhaseI and PhaseII highlights the excellent similar performance over the acceptance of each detector for the two conditions.

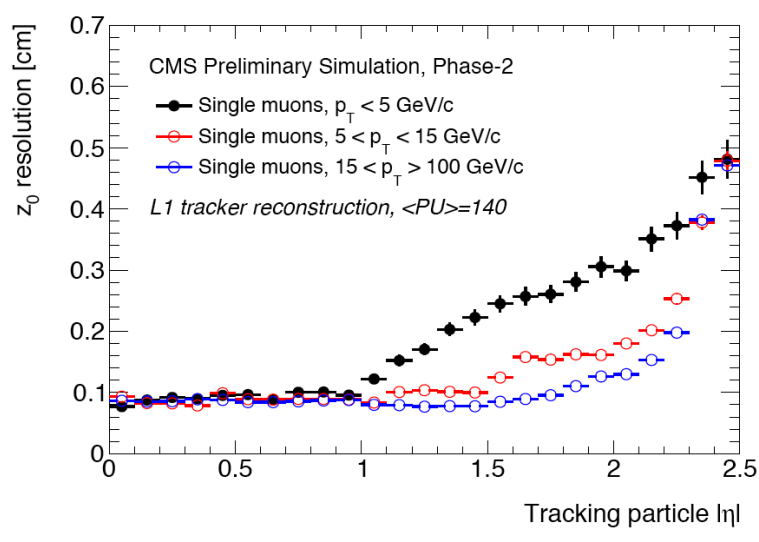

(a)

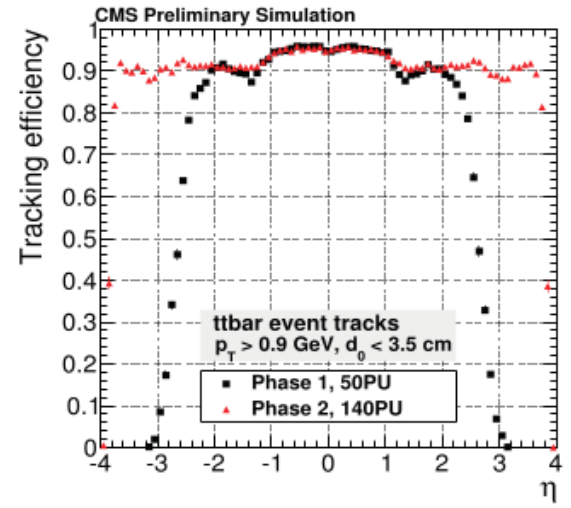

(b)

Figure 6: (a) Impact parameter resolution as a function of $|\eta|$ reconstructed with the tracklet L1 approach for different sub-set of muons. (b) Track reconstruction efficiency as a function of $\eta$ for the Phase-I detector without aging at $50 \mathrm{PU}$ (black) and the Phase-II detector at $140 \mathrm{PU}$ (red) for charged particles from $t \bar{t}$ events with $p_{\mathrm{T}}>0.9 \mathrm{GeV}$ and originating within $3.5 \mathrm{~cm}$ of the beam line (in the transverse direction).

\section{Conclusions \& Outlook}

CMS has developed sophisticated tracking algorithms for RunI to reconstruct the protonproton collision data provided by the CMS silicon tracker. In this paper, a brief description was 
given, and the excellent performance has been shown. In the preparation for the LHC RunII, CMS has faced the challenges lying ahead with a well defined strategy that both reduces the combinatorics and improves the reconstruction performance of specific physics objects. The resulting timing and the performance is comparable to or better than in RunI.

Looking further into the future, a completely new tracker will be installed before LHC enters the high-luminosity phase, in order to provide a satisfactory performance under the extreme conditions at the HL-LHC. The design of the new tracker is well advanced, and its performance in the L1 track trigger has been extensively studied. In the current version of the offline reconstruction software, the full capability of the new strip tracker is not yet exploited. Some possibilities are under study, such as the idea of using different algorithms in different iterations, the possibility to use L1 information, or the introduction of new seeding algorithms that use the high quality stubs provided by the stacked modules of the new tracker.

\section{References}

[1] CMS Collaboration, The CMS experiment at the CERN LHC, Journal of Instrumentation, Volume 3, August 2008, doi:10.1088/1748-0221/3/08/S08004.

[2] CMS Collaboration, Description and performance of track and primary-vertex reconstruction with the CMS tracker, JINST 9 (2014) P10009, doi : $10.1088 / 1748-0221 / 9 / 100 /$ P10009.

[3] G. Cerati, Tracking and vertexing algorithms at high pileup, Vertex 2014 Conference Report

[4] CMS Collaboration, CMS Tracking POG Performance Plots for EPS 2015, https : //twiki.cern.ch/twiki/bin/view/CMSPublic/TrackingPoGPlotsEPS2015

[5] CMS Collaboration, Technical Proposal for the Phase-II Upgrade of the CMS Detector, CERN-LHCC-2015-010, https: / / cds . cern. ch/record/2020886. 